\title{
The comb signal and its Fourier transform
}

\author{
Manuel Duarte Ortigueira*,1 \\ Instituto Superior Técnico and UNINOVA, Monte da Caparica, Portugal
}

Received 25 February 1999; received in revised form 6 October 2000; accepted 8 October 2000

\begin{abstract}
In this paper, we study the aperiodic comb signal from the point of view of the Fourier transform. The comb is very important in the theory of ideal sampling. The knowledge of its properties is crucial for the establishment of suitable interpolation schemes. Here, we present sufficient conditions so that the Fourier transform of an aperiodic comb is an aperiodic comb. We use this result to propose: (1) an alternative approach to the definition of an almost periodic signal and its anharmonic Fourier series; (2) a generalisation of the Shannon-Whittakker sampling/reconstruction for the irregular sampling case. Application of this theory to pulse duration modulation and pulse position modulation is also presented. (C) 2001 Elsevier Science B.V. All rights reserved.
\end{abstract}

Keywords: Comb; Anharmonic Fourier Series; Irregular sampling; Almost periodic

\section{Introduction}

The comb signal is one of the most important entities in Signal Processing, because of its connections with Fourier Series (FS) and ideal sampling [8]. The usual comb is a periodic repetition of the Dirac's delta (generalised) function $[10,12]$. As is well known, its Fourier transform (FT) is also a periodic comb [1]. In this paper we will study the FT of the general aperiodic comb signal and formulate conditions to guarantee that its FT is an aperiodic comb, too. To see the importance of this subject, let us consider the following practical situation. One of the objectives in electrocardiogram

\footnotetext{
* Correspondence address: Campus da FCT da UNL, Quinta da Torre, 2825-114, Monte da Caparica, Portugal. Tel.: + 3511-2948520; fax: + 351-1-2957786.

${ }^{1}$ Also with INESC.

E-mail address: mdo@uninova.pt,mdo@eniac.inesc.pt (M.D. Ortigueira).
}

(ECG) processing is the study of the variability of the cardiac frequency. This is usually done from the so-called RR intervals that are the time intervals between peaks of consecutive cardiac beats. These values constitute a time series. We can model the excitation of the heart as a pulse frequency modulation signal. With this, the RR interval signal can be considered as being proportional to the modulating signal. Therefore, we have a signal that is sampled at a non-uniform spacing: the beat peak positions. Let $d_{n}(n=1,2, \ldots, L)$ be a sequence of $\mathrm{RR}$ intervals. Taking 0 as the time origin reference, we define a set of sampling instants:

$t_{n}=t_{n-1}+d_{n} \quad t_{0}=0, n=1,2, \ldots, L$

and a signal, $v(t)$

$v\left(t_{n}\right)=d_{n}$

that is proportional to the modulating signal. In the available commercial systems, the signal $v(t)$ is treated as if it was obtained by uniform sampling. 

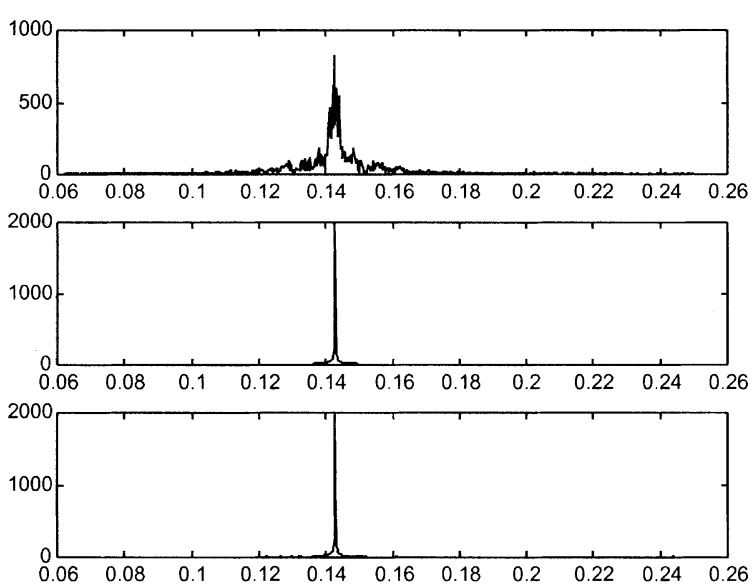

Fig. 1. FFT of a non-uniformly sampled sinusoid (top), FFT of a uniformly sampled sinusoid (middle) and the FT of non-uniformly sampled sinusoid computed through Eq. (1.5) (bottom).

To analyse the error we are making, we took a signal $d_{n}$ obtained from an ECG signal and constructed the sequence of instants, $t_{n}$, through (1.1). We sampled a sinusoid at those instants and at a uniform spacing $n T$, where the sampling interval, $T$, is the mean value of $d_{n}$. For these two signals, we computed their FT by using the FFT. The results are shown in Fig. 1 (top and middle pictures). As it is clear, the use of FFT to compute the FT of the non-uniformly sampled signal is incorrect. To avoid the problem, we assumed that the signal $v(t)$ was ideally sampled by a non-periodic comb

$p(t)=\sum_{-\infty}^{+\infty} \delta\left(t-t_{n}\right)$

obtaining the signal

$v_{\mathrm{s}}(t)=\sum_{-\infty}^{+\infty} v\left(t_{n}\right) \delta\left(t-t_{n}\right)$

that has the following FT:

$V_{\mathrm{s}}(\omega)=\sum_{-\infty}^{+\infty} v\left(t_{n}\right) \mathrm{e}^{-\mathrm{j} \omega t_{n}}$

We used this expression to obtain the spectrum shown at the bottom picture in Fig. 1 that shows a good agreement with the picture in the middle. This fact means that the available approaches to studying the variability of cardiac frequency are intrinsically wrong. These considerations served as motivation for the study we present in this paper.

The problem of non-uniform sampling has received increasing attention due to practical applications in real life. The theory of frames [6] has being the most important tool for dealing with the problem. Here we adopt a more general point of view. We intend to state conditions generalising some of the current results on ideal sampling and reconstruction.

In Section 2, we present the main result of this paper: under stated conditions, the FT of an aperiodic comb is an aperiodic comb [11]. We precise such (sufficient) conditions. The proof is in Appendix A. That result has interesting implications in some well-known fields, as: almost periodic functions and non-uniform sampling. These subjects are treated in Section 3. In Section 4 we present two applications to communication theory: the pulse duration modulation (PDM) and pulse position modulation (PPM) [2]. Most of the mathematical base of the theory is in distribution theory. We present in Appendix B a brief overview of the Axiomatic Theory of Distributions [7,13].

In the following, we represent the sets of integer and real numbers by $Z$ and $R$, respectively. The Dirac's symbol will always be represented by $\delta(t)$.

\section{The FT of a comb}

Consider a set of instants $t_{n}(n=-\infty, \ldots$, $0, \ldots,+\infty)$ assumed to form a, as fast as $n$, increasing sequence such that $t_{ \pm \infty}= \pm \infty$ (for Theorems 2.1 and 2.2, we only need to assume that the sequence increases faster than $\sqrt{|n|}$ ).

Definition 2.1. A comb is a distribution, $c(t)$, defined by

$c(t)=\sum_{-\infty}^{+\infty} \delta\left(t-t_{n}\right)$

Theorem 2.1. The series $\sum_{-\infty}^{+\infty} \delta\left(t-t_{n}\right)$ is convergent.

To prove it, let us introduce the function $s(t)$ given by

$s(t)=\sum_{0}^{+\infty} r\left(t-t_{n}\right)-\sum_{-\infty}^{-1} r\left(-t-t_{n}\right)$ 
where $r(t)$ is the ramp function

$r(t)= \begin{cases}t & t \geqslant 0 \\ 0 & t<0\end{cases}$

It is not hard to see that $s(t)$ is a continuous function. In fact, for every finite $t, s(t)$ is a finite sum of continuous functions. This means that both the series in (2.2) are uniformly convergent. According, to Definition B.3 in Appendix B [7,13], the series (2.1) defining the comb is convergent, because $c(t)$ is the second derivative of $s(t)$, that is a continuous function.

The FT (see Appendix B) of an aperiodic comb is an anharmonic [4] FS

$C(\omega)=\sum_{-\infty}^{+\infty} \mathrm{e}^{-\mathrm{j} \omega t_{n}}$

Theorem 2.2. The series $\sum_{-\infty}^{+\infty} \mathrm{e}^{-\mathrm{j} \omega t_{n}}$ is convergent.

For proof, consider the Definition B.9 and the series

$P(\omega)=\sum_{\substack{-\infty \\ n \neq 0}}^{+\infty} \frac{1}{t_{n}^{2}} \mathrm{e}^{-\mathrm{j} \omega t_{n}}$

As $|P(\omega)|<\sum_{-\infty, n \neq 0}^{+\infty} 1 / t_{n}^{2}$ that is a convergent series, since its terms converge to zero faster than $1 /|n|\left(t_{n}(n=-\infty, \ldots, 0, \ldots,+\infty)\right.$ is assumed to form a, as fast as $n$, increasing sequence), the series in (2.5) converges uniformly. $P(\omega)$ is defined by a uniformly convergent series and so it is a continuous function. Its second derivative is $C(\omega)$ and then the series $\sum_{-\infty}^{+\infty} \mathrm{e}^{-\mathrm{j} \omega t_{n}}$ is convergent.

As is well known, the FT of a periodic comb is a periodic comb. We are looking for a generalisation of this result to the non-periodic case. According, to what we just wrote above, we are looking for conditions that ensure that the series (2.4) represents a comb. Let us introduce some generality by using the comb

$C(\omega)=2 \pi \sum_{-\infty}^{+\infty} C_{n} \delta\left(\omega-\omega_{n}\right)$

where $\omega_{n}$ is a, as fast as $n$, increasing sequence, for now. Its $\mathrm{FT}^{-1}$ is the anharmonic FS

$c(t)=\sum_{-\infty}^{+\infty} C_{n} \mathrm{e}^{-\mathrm{j} \omega_{n} t}$
We assume that the sequence $C_{n}$ is bounded (but not necessarily convergent!) and that, to ensure realness of $c(t), C_{n}^{*}=C_{-n}$ and $\omega_{-n}=-\omega_{n}$.

$c(t)=1+2 \sum_{1}^{+\infty}\left|C_{n}\right| \cos \left(\omega_{n} t+\varphi_{n}\right) \quad \varphi_{n}=\arg \left(C_{n}\right)$.

Again, the conditions we are looking for must ensure that (2.7) represents the comb (2.1). If these conditions exist, (2.7) will be a Fourier (nonharmonic) series associated to (2.1) and (2.4) a Fourier series associated to (2.6).

$\sum_{-\infty}^{+\infty} \delta\left(t-t_{n}\right) \sim \sum_{-\infty}^{+\infty} C_{n} \mathrm{e}^{-\mathrm{j} \omega_{n} t}$.

and

$2 \pi \sum_{-\infty}^{+\infty} C_{n} \delta\left(\omega-\omega_{n}\right) \sim \sum_{-\infty}^{+\infty} \mathrm{e}^{-\mathrm{j} \omega t_{n}}$.

According to the above theorems, all these four series are convergent.

To go further introduce a sequence of intervals $I_{n}^{1}$ and $I_{n}^{2}(n=-\infty, \ldots, 0, \ldots,+\infty)$ defined by

$\left.\left.I_{n}^{1}=\right] n-\frac{1}{4}, n+\frac{1}{4}\right], \quad n \in Z$

and

$\left.\left.I_{n}^{2}=\right] n+\frac{1}{4}, n+\frac{3}{4}\right], \quad n \in Z$,

where $] \cdot]$ stands for a left-open right-closed interval.

Definition 2.2. We define an almost linear sequence (ALS) [4], $T_{F}$, with uniform density equal to $|F|$ [5], as a strictly increasing sequence satisfying:

$T_{F}=\left\{t_{n:} F t_{n} \in I_{n}^{1} n \in Z, F \in R\right\}$

or

$T_{F}=\left\{t_{n:} F t_{n} \in I_{n}^{2} n \in Z, F \in R\right\}$

(see Fig. 2).

In other words, to form an ALS with density $F$ we only have to pick one point and only one in 


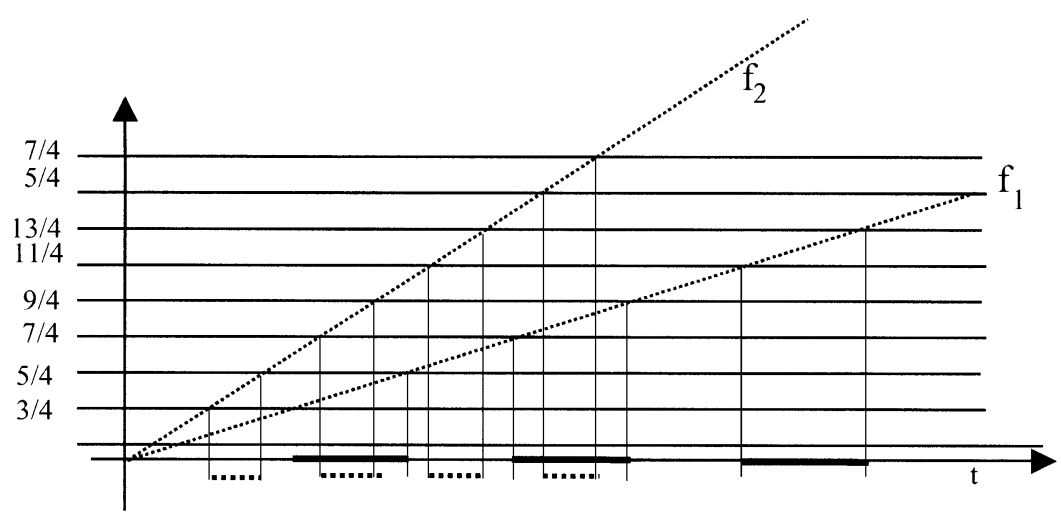

Fig. 2. Definition of (2.11) intervals for two different values of the density.

each interval of a given set: e.g. $\left.t_{n} \in\right](n-1 / 4) /$ $F,(n+1 / 4) / F] n \in Z$, for type 1 ALS. For the other set, $I_{n}^{2}$, we would obtain type 2 ALS.

This is a more precise definition than "relatively dense" and has a wider generality than the similar definition given by Davis [4]. ${ }^{2}$ It is clear that, according to this definition, all the $t_{n}$ can be written as

$t_{n}=n . T+\tau_{n} \quad T=1 / F \quad n \in Z$

in type 1 case and

$t_{n}=(n+1 / 2) \cdot T+\tau_{n} \quad T=1 / F \quad n \in Z$

in type 2 case, with

$0 \leqslant\left|\tau_{n}\right|<1 /(4 F)=T / 4$.

If all the $\tau_{n}$ are zero, the $t_{n}$ sequence will be uniform $t_{n}=n T=n / F$. In the following we will assume that the sequence of $\tau_{n}$ has a zero mean value (if the $\tau_{n}$ sequence were not zero mean valued, $t_{n}$ could be written as $t_{n}=n T+\tau_{0}+\tau_{n}$, corresponding to a sliding of the whole sequence. So, we do not lose generality with this assumption). In Fig. 2 we show how the intervals are defined for different values of $F\left(f_{1}>f_{2}\right)$.

From (2.15) or (2.16), we conclude that the minimum distance between consecutive elements in an

\footnotetext{
${ }^{2}$ We tried, but failed to prove the conjecture: "every ALS with density $F$ is a sub-sequence of all the ALS with density $k F, k$ any integer". In this case the proof of Theorem 2.3 would be simpler.
}

ALS is equal to $1 / 2 F$, while the maximum distance is $3 / 2 F$. This means that every strictly increasing sequence with minimum and maximum distances equal, respectively, to $\Delta_{m}$ and $\Delta_{M}$ with $\Delta_{M} \leqslant 3 \Delta_{m}$ is an ALS. We compute $T$ from a given sequence, $t_{n}$, by adjusting to it a "stair" function:

$\tau_{n}=t_{n}-n T$

and forcing $\tau_{n}$ to have zero mean. As $n T$ is an odd function, we force both sequences (corresponding to $n>0$ and $n<0$ ) to be approximated by a stair function. Consider the expressions

$\lim _{N \rightarrow \infty} \sum_{1}^{N}\left(t_{n}-n T\right)=0 \rightarrow T=\lim _{N \rightarrow \infty} \frac{2}{N(N+1)} \sum_{1}^{N} t_{n}$

and

$$
\begin{aligned}
\lim _{N \rightarrow \infty} & \sum_{1}^{N}\left(-t_{n}-n T\right)=0 \rightarrow T \\
= & -\lim _{N \rightarrow \infty} \frac{2}{N(N+1)} \sum_{1}^{N} t_{-n} .
\end{aligned}
$$

The average of the two is

$$
T=\lim _{N \rightarrow \infty} \frac{2}{N(N+1)} \sum_{1}^{N}\left(t_{n}-t_{-n}\right) .
$$

Definition 2.3. We define an almost periodic comb as a comb defined on an almost linear sequence.

Theorem 2.3. If $t_{n}$ is an almost linear sequence, there is an almost linear odd sequence $\omega_{k}=-\omega_{-k}$ such 


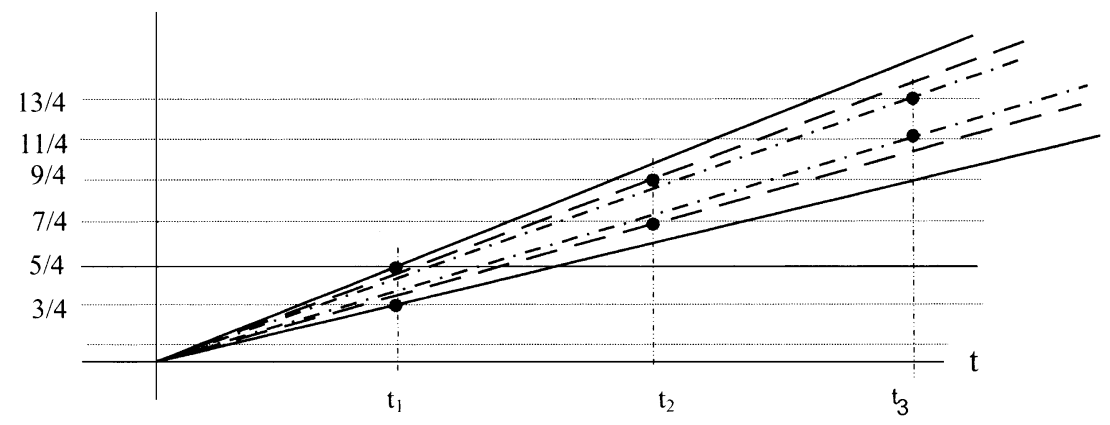

Fig. 3. Sequence of intervals defined by (2.23) to compute the almost harmonics from the set of instants.

that

$\mathrm{FT}\left[\sum_{-\infty}^{+\infty} \delta\left(t-t_{n}\right)\right]=2 \pi \sum_{-\infty}^{+\infty} C_{n} \delta\left(\omega-\omega_{n}\right)$,

where the $C_{n}$ coefficients form a bounded sequence and are given by

$C_{k}=\frac{1}{T} \lim _{N \rightarrow \infty} \frac{1}{2 N+1} \sum_{n=-N}^{N} \mathrm{e}^{-\mathrm{j} \omega_{k} t_{n}}$.

The proof is presented in Appendix A.

Now, it is important to see how to compute the values of $\omega_{k}$ from the given $t_{n}$. We proceed recursively for each set of horizontal strips. Consider Fig. 3. The vertical line passing at $t_{n}$ defines with the boundaries of the horizontal strips two points. These, together with the origin define two straight lines with slopes:

$f_{k, n}^{\mathrm{s}}=\frac{k \cdot n+1 / 4}{t_{n}}$

and

$f_{k, n}^{\mathrm{i}}=\frac{k \cdot n-1 / 4}{t_{n}}$.

For $n=1,2,3, \ldots$ compute those slopes and the points $K_{k, n}^{\mathrm{i}}=f_{k, n}^{\mathrm{i}} \cdot t_{n+1}$ and $K_{k, n}^{\mathrm{s}}=f_{k, n}^{\mathrm{s}} \cdot t_{n+1}$. Begin by computing the intersection of the vertical line passing at $t_{1}$ with the strip centred at $k=1$. Let $k$ integer and $k \in\left[K_{k, n}^{\mathrm{i}}, K_{k, n}^{\mathrm{s}}\right]$. If $k$ is odd repeat the procedure for the next $n$. The sequence of slopes define a sequence of intervals, $I_{k, n}$ :

$\left.I_{k, n}=\right] f_{k, n}^{s}, f_{k, n}^{i}[n=1,2,3, \ldots$.
If this sequence converges, it does so to a degenerate interval $] F_{1}, F_{1}$ [, where $F_{1}$ is the common limit of the sequences defined by (2.21) and (2.22) and it is the frequency of the 1st "almost harmonic". If $k$ is even, ${ }^{3}$ stop the procedure and go to another "almost harmonic" frequency. For the second "almost harmonic", intersect the vertical line passing at $t_{1}$ with the strip centred at $\frac{1}{2}$. Repeat the procedure.

As it is clear the previous procedure can be used to compute other frequencies (almost harmonic frequencies) and we can also conclude that the set of these frequencies is numerable.

\section{Consequences}

Let $x_{\mathrm{b}}(t)$ a signal with FT $X_{\mathrm{b}}(\omega)$ and $t_{n}$ an ALS. Let us assume that $X_{\mathrm{b}}(\omega)$ is a bounded function. We define an almost periodic function, $x(t)$, as the generalised function resulting from convoluting $x_{\mathrm{b}}(t)$ with $c(t)$ given by (2.1). Attending to the properties of the $\delta$ function, we can write

$x(t)=\sum_{n=-\infty}^{\infty} x_{\mathbf{b}}\left(t-t_{n}\right)$

By the use of Eq. (2.19) we conclude that $x(t)$ is represented by the anharmonic FS [3,9]:

$x(t)=\sum_{-\infty}^{+\infty} x_{n} \mathrm{e}^{\mathrm{j} \omega_{n} t}$

\footnotetext{
${ }^{3}$ The even sequence would correspond to a type 2 ALS, that is not suitable here, since $\omega_{0}=0$ (see Fig. 3).
} 
with

$X_{n}=C_{n} \cdot X_{\mathrm{b}}\left(\omega_{n}\right)$

However, using (2.1) and the definition of FT, we obtain

$$
\begin{aligned}
x_{n} & =\left\langle c(t) \mathrm{e}^{-\mathrm{j} \omega_{n} t}\right\rangle \int_{-\infty}^{+\infty} x_{\mathrm{b}}(\tau) \mathrm{e}^{-\mathrm{j} \omega_{n} \tau} \mathrm{d} \tau \\
& =\lim _{\Theta \rightarrow \infty} \frac{1}{2 \Theta} \int_{-\Theta}^{+\Theta} \int_{-\Theta}^{+\Theta} c(t) x_{\mathrm{b}}(s-t) \mathrm{e}^{-\mathrm{j} \omega_{n} s} \mathrm{~d} s \mathrm{~d} t
\end{aligned}
$$

or, by changing the integration orders and noting that the convolution of $c(t)$ with $x_{\mathbf{b}}(t)$ is $x(t)$ :

$X_{n}=\lim _{\Theta \rightarrow \infty} \frac{1}{2 \Theta} \int_{-\Theta}^{\Theta} x(s) \mathrm{e}^{-\mathrm{j} \omega_{n} s} \mathrm{~d} s$

that is the usual way of computing the Fourier coefficients associated with an almost periodic function [3,9]. Therefore, an almost periodic function is an almost periodic repetition of a basic function.

It is important to remark that the reduction of (3.2) to a trigonometric polynomial is obtained only for signals, $x_{\mathrm{b}}(t)$, which are band-limited or have a FT with nulls at the $\omega_{n}$ for $n>N_{0} \in Z^{+}$(since $X_{n}=0$ ).

The dual problem leads to the ideal sampling. Defining a signal $x_{\mathrm{p}}(t)$ by

$x_{\mathrm{p}}(t)=x(t) \cdot c(t)$

we obtain after using Eq. (2.19)

$X_{\mathrm{p}}(\omega)=\sum_{n=-\infty}^{\infty} C_{n} X\left(\omega-\omega_{n}\right)$

generalising the well-known result. Similarly, insert (2.1) in (3.5) and transforming, we obtain, immediately

$X_{\mathrm{p}}(\omega)=\sum_{n=-\infty}^{\infty} x\left(t_{n}\right) \mathrm{e}^{-\mathrm{j} \omega t_{n}}$

that shows that $X_{\mathrm{p}}(\omega)$ is an almost periodic function. If $x(t)$ is a band-limited signal, $\sigma$-BL, we can recover $x(t)$ from $X_{\mathrm{p}}(\omega)$ by ideal low-pass filtering provided that $\omega_{1} \geqslant 2 \sigma$. So, let $\sigma \leqslant W \leqslant \omega_{1}$. As, from (2.20) and (a.9), $C_{0}=\Omega / 2 \pi$, the filter must have a gain equal to $2 \pi / \Omega$ and its output is easily obtained from (3.7)

$$
\begin{aligned}
x(t) & =\sum_{n=-\infty}^{\infty} x\left(t_{n}\right) \frac{1}{\Omega} \int_{-W}^{W} \mathrm{e}^{j \omega\left(t-t_{n}\right)} \mathrm{d} \omega \\
& =\sum_{n=-\infty}^{\infty} x\left(t_{n}\right) \frac{\sin \left[W\left(t-t_{n}\right)\right]}{(\Omega / 2)\left(t-t_{n}\right)}
\end{aligned}
$$

that is the generalisation of the Shannon-Whittaker cardinal series. In particular, putting $t=k T$ and $t_{n}=\left(n+\alpha_{n}\right) T$ and noting that $T . \Omega=2 \pi$, we obtain

$x(k T)=\sum_{n=-\infty}^{\infty} x\left(t_{n}\right) \frac{\sin \left[\pi \cdot \gamma\left(k-n-\alpha_{n}\right)\right]}{\pi\left(k-n-\alpha_{n}\right)}$

where

$\gamma=\frac{W T}{\pi}=\frac{2 W}{\Omega} \leqslant 1$.

Eq. (3.9) allows us to obtain the values of a signal on a uniform time spacing from an irregular one. If one puts $t=t_{k}=\left(k+\alpha_{k}\right) T(k \in Z)$ and $t_{n}=n T$ in (3.8), and notes that the sine function is even, we have

$x\left(t_{k}\right)=\sum_{n=-\infty}^{\infty} x(n T) \frac{\sin \left[\pi \cdot \gamma\left(k-n-\alpha_{n}\right)\right]}{\pi\left(k-n-\alpha_{n}\right)}$.

A comparison of Eqs. (3.9) and (3.11) leads us to conclude, that under the described circumstances the sine functions appearing in those equations are orthogonal.

\section{Applications to pulse modulation}

In the following, we are going to study two important cases that fall inside the theory we presented in previous sections. We will present the expressions for the modulated signals in pulse duration modulation (PDM) and in pulse position modulation (PPM) [13].

We will consider the PDM signal with trailingedge modulation of the pulse duration. The modulating signal dependence is on the location, $t_{k}$, $k=-\infty, \ldots,+\infty$, of the pulse edge. According 
to the generalisation of Nyquist criterion stated in (A.11), the sampling instants will be given by (2.15) or (2.16) with $F$ equal to twice the bandwidth of the modulating signal, $x(t)$. In [13], it is assumed $t_{k}-k T \ll T$, that is a too restrictive relation according to what we just saw. To correctly represent the PDM signal, we found better to use (2.16), instead (2.15). We consider that each pulse begins at $k T$ and lasts at

$t_{k}=k T+T / 2+\tau_{n}$,

where

$\tau_{n}=\mu . x(t)$

with $|x(t)| \leqslant 1$ and $0<\mu<T / 4$. Without losing generality, $x(t)$ is assumed to have a null mean.

It is not a simple task to obtain directly the expression of the modulated signal, $s_{\mathrm{PDM}}(t)$. This is a sequence of rectangular width-variant pulses. However, it is very easy to write its derivative:

$s_{\text {PDM }}^{\prime}(t)=\sum_{-\infty}^{+\infty} \delta(t-n T)-\sum_{-\infty}^{+\infty} \delta\left(t-t_{n}\right)$

that can be represented by the Fourier series:

$s_{\text {PDM }}^{\prime}(t)=\frac{1}{T} \sum_{-\infty}^{+\infty} \mathrm{e}^{\mathrm{j}(2 \pi / T) n t}-\sum_{-\infty}^{+\infty} C_{n} \mathrm{e}^{\mathrm{j} \omega_{n} t}$

easily obtained from (2.9). The Fourier coefficients are computed from (2.20). Computing the primitives of both series in (4.4), we obtain the expression of the PDM signal:

$S_{\mathrm{PDM}}(t)=S_{0}+\sum_{n \neq 0} \frac{1}{\mathrm{j} 2 \pi n} \mathrm{e}^{\mathrm{j}(2 \pi / T) n t}-\sum_{n \neq 0} \frac{C_{n}}{\mathrm{j} \omega_{n}} \mathrm{e}^{\mathrm{j} \omega_{n} t}$

where $S_{0}$ is equal to $1 / 2$, that is the rectangle mean width.

The PPM signal is obtained from the PDM signal, by generating fixed width $(\tau<T)$ rectangular pulses at the end of the PDM pulses. Thus, it is a simple task to observe that

$s_{\mathrm{PDM}}^{\prime}(t)=\sum_{-\infty}^{+\infty} \delta\left(t-t_{n}\right)-\sum_{-\infty}^{+\infty} \delta\left(t-t_{n}-\tau\right)$.

Proceeding as with the PDM signal, we obtain

$$
S_{\mathrm{PDM}}(t)=\frac{\tau}{T}+\sum_{n \neq 0} \frac{C_{n}}{\mathrm{j} \omega_{n}} \mathrm{e}^{\mathrm{j} \omega_{n} t}-\sum_{n \neq 0} \frac{C_{n}}{\mathrm{j} \omega_{n}} \mathrm{e}^{\mathrm{j} \omega_{n} \tau} \mathrm{e}^{\mathrm{j} \omega_{n} t}
$$

or

$S_{\text {PDM }}(t)=\frac{\tau}{T}+\sum_{n \neq 0} \frac{C_{n}}{\mathrm{j} \omega_{n}}\left[1-\mathrm{e}^{\mathrm{j} \omega_{n} \tau}\right] \mathrm{e}^{\mathrm{j} \omega_{n} t}$

that is the Fourier series associated to the PPM signal.

\section{Conclusions}

In this paper, we studied the aperiodic comb signal and its FT. We showed how we can guarantee that the FT of an aperiodic comb is an aperiodic comb. We used this result to propose an alternative approach to the definition of an almost periodic signal and computed its anharmonic Fourier series. The original first goal of this theory was the generalisation of the Shannon-Whittakker sampling/ reconstruction for the irregular sampling case was also presented. Based on the previous results we presented an application to pulse communication by proposing exact expressions for pulse duration modulation and pulse position modulation signals.

\section{Appendix A. Proof of Theorem 2.3}

Before going into the proof, we introduce the long-term average (LTA) [9] or mean value [3], $\langle g\rangle$, of a generalised function, $g(t)$, by

$\langle g\rangle=\lim _{\Theta \rightarrow \infty} \frac{1}{2 \Theta} \int_{-\Theta}^{\Theta} g(t) \mathrm{d} t$.

A more rigorous statement would write

$\langle g\rangle=\lim _{\Theta \rightarrow \infty} \frac{1}{2 \Theta} \int_{-\Theta+\alpha}^{\Theta+\alpha} g(t) \mathrm{d} t$

and the convergence would be uniform in $\alpha$. However, as we work in the field of the generalised functions, this subject is not important. With (A.1), it is easy to show that

$\left\langle\mathrm{e}^{\mathrm{j} \omega_{n} t} \mathrm{e}^{-\mathrm{j} \omega_{k} t}\right\rangle=\left\{\begin{array}{lll}0 & \text { if } & \omega_{n} \neq \omega_{k}, \\ 1 & \text { if } & \omega_{n}=\omega_{k} .\end{array}\right.$ 
For proof of Theorem 2.3, assume that (2.19) is valid. Computing the inverse FT of both members, we obtain

$\sum_{-\infty}^{+\infty} \delta\left(t-t_{n}\right)=\sum_{-\infty}^{+\infty} C_{n} \mathrm{e}^{\mathrm{j} \omega_{n} t}$.

Multiplying both members of (A.3) by $\mathrm{e}^{-\mathrm{j} \omega_{n} t}$ and computing the LTA, we obtain

$\left\langle\sum_{-\infty}^{+\infty} \delta\left(t-t_{n}\right) \mathrm{e}^{-\mathrm{j} \omega_{k} t}\right\rangle=\left\langle\sum_{n=-\infty}^{+\infty} C_{n} \mathrm{e}^{\mathrm{j} \omega_{n} t} \mathrm{e}^{-\mathrm{j} \omega_{k} t}\right\rangle$.

Using (A.2) the right-hand side in (A.4) is $C_{k}$, that, by using (A.1) leads to

$C_{k}=\lim _{\Theta \rightarrow \infty} \frac{1}{2 \Theta} \int_{-\Theta}^{\Theta} \sum_{-\infty}^{+\infty} \delta\left(t-t_{n}\right) \mathrm{e}^{-\mathrm{j} \omega_{k} t} \mathrm{~d} t$

that is the formula to compute the Fourier coefficients [3,9]. If the frequencies $\omega_{k} k=1,2, \ldots$, are multiples of a given frequency $\omega_{0}$, we obtain the ordinary (harmonic) FS. Now, write $\Theta=\left(N+\frac{1}{2}\right) T$. Performing the computation, we obtain

$C_{k}=\frac{1}{T} \lim _{N \rightarrow \infty} \frac{1}{2 N+1} \sum_{n=-N}^{N} \mathrm{e}^{-\mathrm{j} \omega_{k} t_{n}}$.

The sequence $C_{n}$ is bounded which guarantees the convergence of the series in (2.9) and (2.10). In fact $\left|C_{n}\right| \leqslant 1 / T$. We are going to give an interpretation to Eq. (A.6).

(a) The signal $\mathrm{e}^{\mathrm{j} \omega_{k} t_{n}}$ is the result of sampling a cisoid $\mathrm{e}^{\mathrm{j} \omega_{k} t}$ in a time spacing that is an ALS. Eq. (A.4) represents the average of those values.

(b) When performing the summation in (A.6) we are adding unitary vectors in the complex plane. The resulting vector is, in general, of finite length, even if we extend the summation to $\infty$. This does not happen if the vectors are collinear, or in a more general situation, if all their extremities lie in the same complex half plane (right or left). If the vectors are collinear, the resulting vector will have a length equal to the sum of the lengths of all the vectors; otherwise, the resulting vector will have a length inferior to the sum of the lengths. Assuming that $t_{n}$ has the form (2.15) or (2.16) with $\tau_{n}$ having zero mean, the length of the resulting vector will be equal to the sum of the real parts of the vectors and so

$C_{k}=\frac{1}{T} \lim _{N \rightarrow \infty} \frac{1}{2 N+1} \sum_{n=-N}^{N} \cos \left(\omega_{k} t_{n}\right)$,

where $\omega_{k} t_{n}=2 \pi f_{k} t_{n}$ and $f_{k} t_{n}$ satisfies (2.13) or (2.14) for all $n, k \in Z$. As the first member in (2.19) is given by (2.4) and using the LTA definition we can write

$1=\lim _{W \rightarrow \infty} \frac{1}{2 W} \int_{-W}^{W} 2 \pi \sum_{-\infty}^{+\infty} C_{n} \delta\left(\omega-\omega_{n}\right) \mathrm{e}^{\mathrm{j} t_{k} \omega} \mathrm{d} \omega$

Now, write $\Theta=\left(N+\frac{1}{2}\right) \Omega$ and perform the computation to obtain

$1=\frac{2 \pi}{\Omega} \lim _{N \rightarrow \infty} \frac{1}{2 N+1} \sum_{k=-N}^{N} C_{k} \mathrm{e}^{\mathrm{j} \omega_{k} t_{n}} \quad n \in Z$

that is valid for every $n \in Z$. Inserting (A.6) into (A.9), we obtain after some manipulation:

$$
\begin{aligned}
1= & \frac{2 \pi}{\Omega T} \lim _{N, M \rightarrow \infty} \frac{1}{(2 N+1)(2 M+1)} \\
& \times \sum_{m=-N}^{N} \sum_{k=-M}^{M} \mathrm{e}^{-\mathrm{j} \omega_{k}\left(t_{m}-t_{n}\right)} \quad n \in Z .
\end{aligned}
$$

According to the considerations we did before concerning the interpretation of the summations as vectors, we conclude that in the $\sum_{m=-N}^{N}$ $\sum_{k=-M}^{M} \mathrm{e}^{-\mathrm{j} \omega_{k}\left(t_{m}-t_{n}\right)}$ only the terms corresponding to $t_{m}=t_{n}$, contribute to the limit. This is not difficult to observe. We have

$$
\begin{aligned}
\left|\sum_{m=-N}^{N} \sum_{k=-M}^{M} \mathrm{e}^{-\mathrm{j} \omega_{k}\left(t_{m}-t_{n}\right)}\right| & \leqslant \sum_{m=-N}^{N} \sum_{k=-M}^{M}\left|\mathrm{e}^{-\mathrm{j} \omega_{k}\left(t_{m}-t_{n}\right)}\right| \\
& =(2 N+1)(2 M+1) .
\end{aligned}
$$

But the terms corresponding to $t_{m}=t_{n}$, contribute with the value 1 independently of $\omega_{k}$. So,

$\sum_{m=-N}^{N} \sum_{k=-M}^{M} 1=(2 N+1)(2 M+1)$

and the other terms add to zero:

$\sum_{\substack{m=-N \\ m \neq n}}^{N} \sum_{k=-M}^{M} \mathrm{e}^{-\mathrm{j} \omega_{k}\left(t_{m}-t_{n}\right)}=0 \quad n \in Z$.

This is a consequence of the fact that $t_{m}-t_{n}=$ $(m-n) T+\tau_{m}-\tau_{n}$ where $\left.\tau_{m}-\tau_{n} \in\right]-\frac{1}{2}, \frac{1}{2}[(m$, $n \in Z, m \neq n)$ has zero mean. This means that the 
corresponding vectors are likely to have their extremities almost uniformly distributed on the unit circle, so adding to zero. It is easy, now, to obtain the important formula

$\frac{2 \pi}{\Omega T}=1$

which is the generalisation of the Nyquist result obtained in the uniform case. Consider (A.9) again, multiply both sides by $\mathrm{e}^{-\mathrm{j} \omega_{m} t_{n}}$, compute the average value and use (A.10)

$$
\begin{aligned}
& \frac{1}{T} \lim _{N \rightarrow \infty} \frac{1}{2 N+1} \sum_{n=-N}^{N} \mathrm{e}^{\mathrm{j} \omega_{k} t_{n}} \\
& \quad=\frac{1}{T^{2}} \lim _{N \rightarrow \infty} \frac{1}{(2 N+1)^{2}} \sum_{k=-N}^{N} C_{k} \sum_{n=-N}^{N} \mathrm{e}^{\mathrm{j}\left(\omega_{k}-\omega_{m}\right) t_{n}} .
\end{aligned}
$$

The LHS is $C_{m}$. So, if $\omega_{k} \neq \omega_{m} \neq 0$ from the second member we conclude that

$$
\lim _{N \rightarrow \infty} \frac{1}{2 N+1} \sum_{n=-N}^{N} \mathrm{e}^{\mathrm{j}\left(\omega_{k}-\omega_{m}\right) t_{n}}=0 \quad k \neq m,
$$

which states orthogonality between two different sinusoids sampled at the same points and is the discrete-time version of (A.2). As $\omega_{k}$ is an ALS sequence

$\omega_{k}=k \Omega+v_{k} \quad k \in Z,\left|v_{k}\right|<\Omega / 4$, and $v_{0}=0$. (A.13)

$\omega_{k}-\omega_{m}=(k-m) \Omega+v_{k}-v_{m}$ with $\left|v_{k}-v_{m}\right|<$ $\Omega / 2$, ensuring that, with $t_{n}$ an ALS, the extremities of vectors in (A.8) are likely to be at any point on the unit circle, leading to a null average of the vectors. Eq. (A.13) fixes only the format of the $\omega_{k}$ sequence; it does not mean that all the corresponding "almost harmonics" exist, because the respective coefficients may be zero. For example, consider the "extreme" sequence $t_{n}=n T \pm T / 4$, with the signal \pm being selected randomly or irregularly. In this case, the first almost harmonic has a null coefficient.

\section{Appendix B. On the axiomatic theory of distributions}

\section{B.1. Motivation}

In the following, we will present a brief overview on the axiomatic theory of distributions. Although this theory was developed by Prof. J. Sebastião and Silva [13] in the 1950s, it remains almost unknown in the engineering community. However, in our opinion, it is the most intuitive and direct of the approaches to distributions. Davis [5] proposed an approach that we consider to be a particular case of the theory we are going to present. The idea underlying the axiomatic theory of distributions is very simple: "enlarge the class of functions in order to make possible to differentiate indefinitely any continuous function".

To begin let $I$ be an interval in $R, C(I)$ the set of continuous functions on $I$ and $C^{p}(I)$ the set of functions $p$ times continuously derivable in the usual sense. We represent by $D$ the operator $\mathrm{d} / \mathrm{d} t$. So, a function $g \in C^{p}(I)$ can be represented by

$f=D^{p} g$,

where $f \in C(I)$. The right inverse operator of $D$ (primitivation operator) is represented by $S$ and satisfies

$S^{p} f=g$

with

$S f=\int_{\mathrm{I}} f(\tau) \mathrm{d} \tau$.

As the primitive of a given function is defined aside a constant, the set $C^{1}(I)=\{S f+K: f \in C(I), K$ constant $\}$ is contained in $C(I): C(I) \supset C^{1}(I)$. Defining the powers of the operator $S$ by the recursion: $S^{n} f=S\left(S^{n-1}\right) f$, with $S^{0} f=f$ and denoting by $P_{n-1}$ the set of $(n-1)$ th degree polynomials, we will have

$C^{n}(I)=\left\{S^{n} f+p: f \in C(I)\right.$ and $\left.p \in P_{n-1}\right\}$,

where $C^{n}(I)$ is the set of the $n$ times continuously differentiable functions in $R$. Putting, now, $C^{\infty}(I)$ as the intersection of all the $C^{n}(I)(n=1, \ldots, \infty)$, we conclude that

$$
\begin{aligned}
C(I) \supset C^{1}(I) \supset \cdots & \supset C^{n-1}(I) \supset C^{n}(I) \\
& \supset C^{n+1}(I) \supset \cdots \supset C^{\infty}(I) .
\end{aligned}
$$

For every $n \in N$, the derivation can be viewed as an application of $C^{n+1}$ in $C^{n}$. Its generalisation 
consists essentially in prolonging the previous sequence to the left in order to obtain

$$
\begin{aligned}
& C_{\infty}(I) \supset \cdots \supset C_{n+1}(I) \supset C_{n}(I) \\
& \quad \supset C_{n-1}(I) \supset \cdots \supset C_{1}(I) \supset C(I) .
\end{aligned}
$$

We represent by $C_{\infty}(I)$ the reunion of all the $C_{n}(I)(n=1, \ldots, \infty)$ say, the set of all the "functions" $\varphi$ that result from the repeated application of the operator $D$ to $f \in C(I)$.

Definition B.1. The elements $\varphi \in C_{\infty}(I)$ such that

$$
\varphi=D^{p} f \quad p \in N_{0}, \quad f \in C(I),
$$

we will call generalised functions or distributions $(G F)$.

Therefore, any distribution is a derivative of a continuous function. Example: the second-order derivative of the function $x(t)=|t|$ is twice the Dirac impulse.

\section{B.2. The axioms}

With this definition, any continuous function is a distribution. These considerations lead us to the intuitive notion of distribution. However, we need a correct formal framework for the GF theory. For this, we introduce two primitive terms: distribution (or GF) and derivative (generalised). The precise meaning of these terms is established when constructing the referred formal framework. This is based in four axioms, which will be presented, interpreted and their consequences discussed.

Axiom B.1. If $f \in C(I)$, then $f \in C_{\infty}(I)$

Thus, $C_{\infty}(I)$ is a nonempty set, since it contains, at least, the continuous functions.

Axiom B.2. For each $f \in C_{\infty}(I)$, there is an element $D f \in C_{\infty}(I)$ such that if $f$ has derivative, $f^{\prime} \in C_{\infty}(I)$ in the usual sense, then $D f$ coincides with $f^{\prime}$.

This is a guarantee of infinite differentiability of any GF. This means that, if $f$ is a continuous function, $D^{\mathrm{n}} f$ will represent a distribution. There exists, then, an application from $C \times N_{0}$ into $C_{\infty}(I)$ that associates a distribution $\varphi=D^{n} f$ to each pair $(f, n)$ [12]. To the least natural number in these conditions, we call degree of the distribution. So, zero degree GF are the continuous functions; the Heaviside's unit step has degree 1 and Dirac's impulse has degree 2.

Axiom B.3. For each $\varphi \in C_{\infty}(I)$ there is a $n \in N_{0}$ and a function $f \in C(I)$ such that

$\varphi=D^{n} f$.

We conclude that any distribution is defined by sets of pairs $(f, n)$. For example, the Dirac's delta function can be defined by the pairs $(r, 2)$ and $(u, 1)$, where $r$ and $u$ are the ramp and unit step functions.

Axiom B.4. If $n \in N_{0}$ and $f$ and $g \in C(I)$, then we have $D^{n} f=D^{n} g$ if and only if $f-g$ has the form $f-g=P_{n-1}(t)$, where $P_{n-1}$ represents a polynomial in $t$ with degree less than $n$.

This axiom specifies what one understands by equality of generalised functions (FG), or, the conditions such that two pairs $(f, n)$ and $(g, m)$ represent the same GF; for example if $f(t)=t^{3} u(t)$ the pairs $(f, 2),\left(f^{\prime}, 1\right)$ and $\left(f^{\prime \prime}, 0\right)$ represent the same distribution.

Consider the simple case of two continuous functions defined in $R$. In this case, $D f=f^{\prime}$ and $D g=g^{\prime}$, $D f=D g$ being equivalent to $(f-g)^{\prime}=0$, or, $f-g=$ constant in $R$. Axiom 4 corresponds to accept and generalise this fact. As $D^{n} f=D^{n+m}\left(S^{m} f\right)$ and $D^{m} g=D^{n+m}\left(S^{n} g\right)$, we conclude that $D^{n} f=D^{m} g$, if and only if $S^{m} f-S^{n} g=P_{n+m-1}$.

To finish these considerations relative to the axiomatic it is convenient to say that we would show that it must be compatible and categorical. We do not do it because it is beyond the objectives of this work.

Having this axiomatic in hands, it is not difficult, now, to show that, with suitable definitions of sum of distributions and multiplication by a constant, the space of distributions is a linear vectorial space. This means that, particularly, the sum of two GF is a GF and the addition enjoys the usual properties. The same does not happen with multiplication. In 
fact, it is not possible to define product of two GF in order to guarantee that it enjoys the usual properties (namely, to be associative, to satisfy the product derivative rule and to coincide with usual product when both factors are continuous functions. We do not go further (see [7]).

\section{B.3. Sequences and series of distributions}

We are going to generalise the notions of limit of a sequence in order to introduce the notion of series.

Definition B.2. Let $f_{n}$ be a sequence of GF. We will say that $f_{n}$ converges to $f$ in $C_{\infty}(I)$ if and only if there exist $p \in N, F$ and $F_{n} \in C(I)$ such that we have $D^{p} F_{n}=f_{n}, D^{p} F=f$ and that $F_{n}$ converges uniformly to $F$ in $I$.

With this definition, we are able to define sum of a series of distributions.

Definition B.3. Let $f_{n}$ be a sequence of distributions in $I$. We say that the series $\sum_{1}^{\infty} f_{n}$ is convergent in $C_{\infty}$ if and only if the sequence of partial sums $g_{k} \sum_{1}^{k} f_{n}$ is convergent.

If the series is bilateral: $\sum_{-\infty}^{+\infty} f_{n}$, it is convergent, if the series $\sum_{1}^{\infty} f_{n}$ and $\sum_{-\infty}^{-1} f_{n}$ are both convergent. The sum of the series is obtained by adding the sums of the two series.

\section{B.4. Fourier transform of distributions}

Let $\varphi$ be a GF in $R$. We define the primitive of $\varphi$ to any GF $\Phi$ such that

$D \Phi=\varphi$.

The integral $\int_{a}^{b} \varphi(t) \mathrm{d} t$ is defined by the generalised Barrow formula:

$\int_{a}^{b} \varphi(t) \mathrm{d} t=\Phi(b)-\Phi(a)$.

An integral exists or is convergent if and only if both limits exist. It can be proved that if a GF is integrable in $R$, then $\varphi(t)=\mathrm{O}\left(t^{-1}\right)$ when $t \rightarrow \infty$. On the other hand, if there exist $\alpha<-1$ such that
$\varphi(t)=\mathrm{O}\left(t^{\alpha}\right)$ when $t \rightarrow \infty$ then $\varphi$ is integrable in $R$ [7].

Similar to the usual procedure, we define FT of a generalised function $\varphi(t)$ as being the function $\Phi(\omega)$ such that

$\Phi(\omega)=\int_{R} \varphi(t) \mathrm{e}^{-\mathrm{j} \omega t} \mathrm{~d} t$

if the integral exists.

Definition B.4. The integral $\int \varphi(t, \tau) \mathrm{d} \tau$ is said to be convergent in $R$ if and only if there exists a primitive $\psi$ of $\varphi$ relatively to $\tau$ convergent in $R$ when $\tau \rightarrow \infty$.

We will write then $\int \varphi(t, \tau) \mathrm{d} \tau=\Psi(t,+\infty)$ $-\Psi(t,-\infty)$ in $R$. In particular, it can be proved that

(a) if $\varphi$ is summable in $R, \operatorname{FT}[\varphi]$ exists and it is a continuous bounded function in $R$.

(b) If $\varphi$ is a tempered ${ }^{4} \mathrm{GF}$, then its FT exists and it is also a tempered GF.

\section{References}

[1] R. Bracewell, The Fourier Transform and Its Applications, McGraw-Hill Book Company, New York, 1965.

[2] A.B. Carlson, Communication Systems, McGraw-Hill International Editions, New York, 1983.

[3] C. Cordoneanu, Almost Periodic Functions, Wiley Interscience Publishers, New York, 1968.

[4] A. M. Davis, Almost periodic extension of band-limited functions and its application to nonuniform sampling, IEEE Trans. Circuits Systems CAS-33 (10) (October 1986) 933-938.

[5] A.M. Davis, $K_{\infty}$ Generalised functions, Proceedings of the IEEE International Conference on Circuits and Systems, Seattle, Washington, DC, USA, 1995, pp. 1652-1659.

[6] R.J. Duffin, A.C. Schaeffer, A class of nonharmonic Fourier series, Trans. Amer. Math. Soc. 72 (1952) 341-366.

[7] J.C. Ferreira, Introdução à Teoria das Distribuições, Fundação Calouste Gulbenkian, 1990. English version: Introduction to the Theory of distributions, Pitman Monographs and Surveys in Pure and Applied Mathematics, Pitman, London, July 1997.

\footnotetext{
${ }^{4} \mathrm{~A}$ generalised function $\varphi$ is said to be tempered or of polynomial type if and only if $\exists \alpha \in R$ such that $\varphi(t) / t^{\alpha}$ is bounded when $t \rightarrow \infty$.
} 
[8] A.J. Jerri, The Shannon sampling theorem - its various extensions and applications: a tutorial review, Proc. IEEE 65 (11) (November 1977) 1565-1596.

[9] B.M. Levitan, V.V. Zhikov, Almost periodic functions and differential equations, Cambridge University Press, Cambridge, 1982.

[10] M.J. Lighthill, Introduction to Fourier analysis and generalised functions, Cambridge University Press, Cambridge, 1964.
[11] M.D. Ortigueira, What about the FT of the comb signal?, Proceedings of SAMPTA-97, Aveiro, Portugal, 1997, pp. 28-33.

[12] L. Schwartz, Théorie des Distributions, Herman \& Cie, Paris, 1966.

[13] J.S. Silva, The axiomatic theory of the distributions, Complete Works, INIC, Lisbon, 1989. 